\title{
Observation of gamma-ray emission from the Markarian 421 with the LHAASO-WCDA
}

\section{Ran Wang ${ }^{a, *}$ and Min Zha ${ }^{b}$, Cunfeng Feng ${ }^{a}$}

${ }^{a}$ Shandong University, 72, Binhai Road, Jimo, Qingdao, Shandong, China

${ }^{b}$ The Institure of High Energy Physics of the Chinese Academy of Sciences, 19B, Yuquan Road, Shijingshan District, Beijing, China

E-mail: wangran@ihep.ac.cn, zham@ihep.ac.cn, fengcf@sdu.edu.cn

The water Cherenkov detector array (WCDA), sub-array of Large High Altitude Air Shower Observatory (LHAASO), is highly sensitive to VHE gamma-ray and is dedicated to surveying the northern sky $(100 \mathrm{GeV}$ to $30 \mathrm{TeV})$. In this work, the $\mathrm{TeV}$ gamma ray emission in Mrk421 is monitored over period from 2019 July to 2020 February with the first detector array of WCDA. The significance and excess over background of this source are measured within this period and compared with the observation by the satellite-borne experiment Fermi in the GeV range. The evolution of the spectral energy distribution is also investigated by measuring spectral indices at two different flux levels.

$37^{\text {th }}$ International Cosmic Ray Conference (ICRC 2021)

July 12 th - 23rd, 2021

Online - Berlin, Germany

\footnotetext{
${ }^{*}$ Presenter
} 


\section{Introduction}

Mrk421( $\mathrm{z}=0.031)$ is one of the most closet and brightest blazars and is classified as a BL Lac object, a subclass of active galactic nuclei (AGNs). Its broadband radiation covering from ratio to gamma-rays, believed to be the rotating central black hole as a power source to transport energy alone the jets to one or multiple emission regions. The model of the AGNs is shown in Fig. 1(Left).
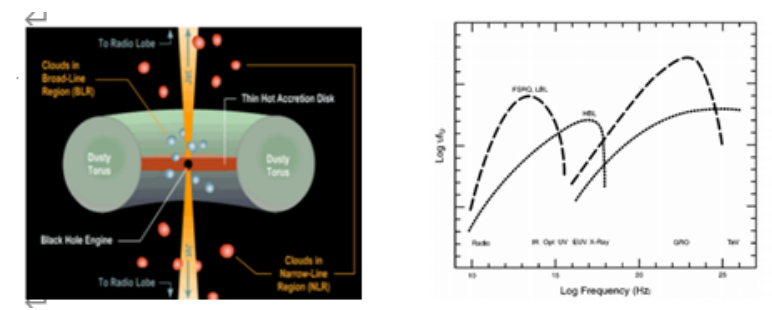

Figure 1: Left:The model structure of Active Galactic Nuclei. Right: Broad-band energy spectrum from radio to TEV gamma-ray

The spectral energy distributions (SEDs) of Mrk421 is double-humped, as shown in Fig.1(Right). The lower energy component, which peak in the optical or X-rays band, believed to be the result of the synchrotron radiation from the relativistic electrons or positrons in the jet, while the origin of the higher energy component has no definite explanation. Depending on the dominating population of accelerated particles being either electrons or protons, there exits two blazar emission models to explain it: the first view is leptonic models, which assumes that the higher energy gamma rays are produced by inverse Compton scattering off the same population of relativistic electrons (SSC) or external electrons (EC). Another view is hadronic models, which assumes that protons are being ceelerated to energies high enough to reach the threshold for photopion production. The more details can be found in the literature[1].

In this paper, we are focusing on the Mrk421, presenting the eight-month light curves, to analysis the flux variations with energies. After brief introduction on the LHAASO-WCDA observatory in Section 2, then we describe the analysis methods in Section 3, including the data selection, background suppression and estimation. We apply the methods to Mrk421 to get the light curve and SED in Section 4.

\section{The WCDA Observatory}

The Water Cherenkov Detector Array (WCDA) is located at the elevation of $4410 \mathrm{~m}$ above sea level in Sichuan Province, China. Covering an area of $78,000 \mathrm{~m}^{2}$. The array consists of three phases as shown in Fig. 2. The lower ponds are WCDA-1 and WCDA-2 from left to right with the size of $150 \mathrm{~m} \times 150 \mathrm{~m}$, the upper one is WCDA-3 with the size of $300 \mathrm{~m} \times 110 \mathrm{~m}$. The whole WCDA consists of 3120 detector units, each unit is partitioned by the black curtains into $5 \mathrm{~m} \times 5 \mathrm{~m}$ with an effective purified water depth up to $4 \mathrm{~m}$. Additionally, each detector cell equipped with two upward-facing PMTs on the bottom at the center of the unit to receive the Cherenkov light produced in water by those secondary particles that are induced by the air showers. The WCDA is designed to focus on the very high energy (VHE) gamma astronomy and survey the TeV sky for steady and transit 
sources, covering energy interval from $100 \mathrm{GeV}$ to $30 \mathrm{TeV}$, with the advantage of a large field of view (FOV), full duty circle, good angular resolution and fine gamma/proton discrimination.
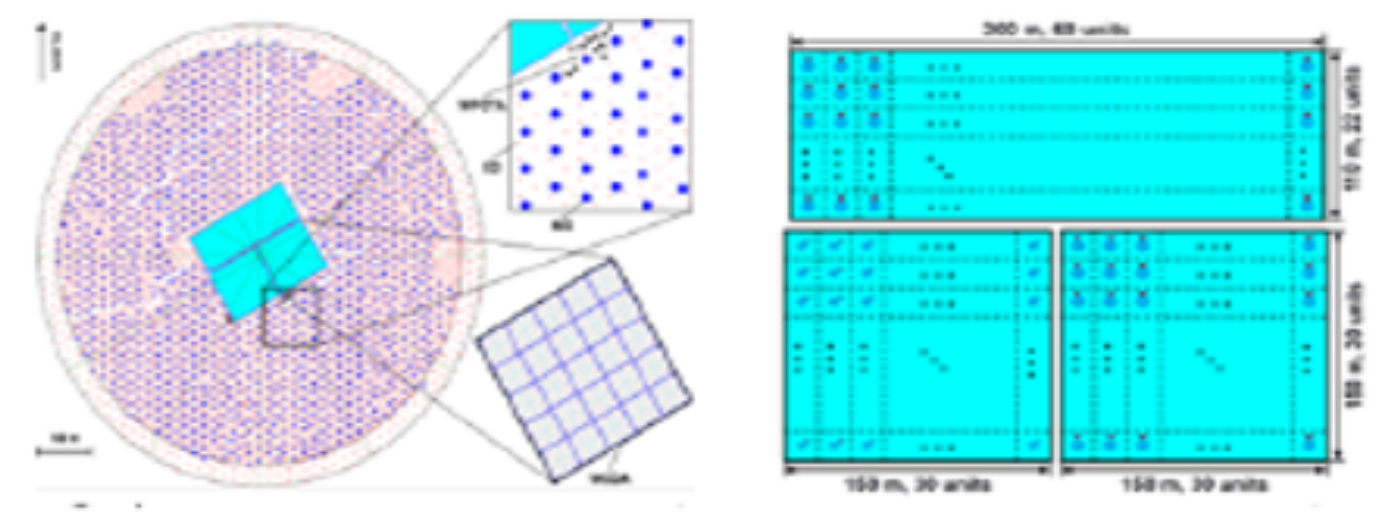

Figure 2: Left:The layout of the LHAASO experiment. LHAASO consists of one square kilometer array (KM2A), Water Cherenkov detector array (WCDA) and wide-field Cherenkov telescope array (WFCTA). Right: Schematic of the WCDA layout.

The observations of very high energy gamma astronomy based on the WCDA will contribute to the observation of the cosmic ray energy spectrum, the limiting model of active galactic nuclei, and the understanding of extreme celestial activity.

\section{Analysis Methods}

\subsection{The Data Selection}

In this paper, we report on the multi-wavelength observations of Mrk421 in the $\mathrm{GeV}$ and $\mathrm{TeV}$ bands, during the eight months period of WCDA and Fermi-LAT common operation time from 2019 July to 2020 February. All events are collected in the period around the Mrk421 direction are grouped into four bins of Nfitc , namely [60,100], [100,200], [200-300] and [300,800]. The criteria for events to be used in the analysis are as follows.

(I) $60 \leq$ Nfitc $<800$.

(II) The Mrk421 was at least 50 degree above the horizon.

(III) The compactness is larger than 15.

\subsection{The gamma/proton separation}

Given that most of the events detected by WCDA are induced by cosmic rays, so maximize the sensitivity to gamma-rays showers through the background suppression is important. Based on the gamma-rays induced showers are pure electromagnetic showers often has a compact, smooth lateral shower profile while the proton-induced showers are more spread out laterally rich with hadronic secondary particles, like pions, muons. One of the most powerful gamma/proton separation techniques is define a parameter named compactness. The compactness is defined as $\mathrm{C}=\mathrm{N}_{h i t} / \mathrm{Max}(\mathrm{Qi} ; \mathrm{r}$ $>\mathrm{Rc}$ ), the method concludes the largest PMT signal beyond Rc from the shower core and the number of triggered PMTs of each event. For WCDA-1, the C $>15$ is optimal to distinguish the muon signal. 

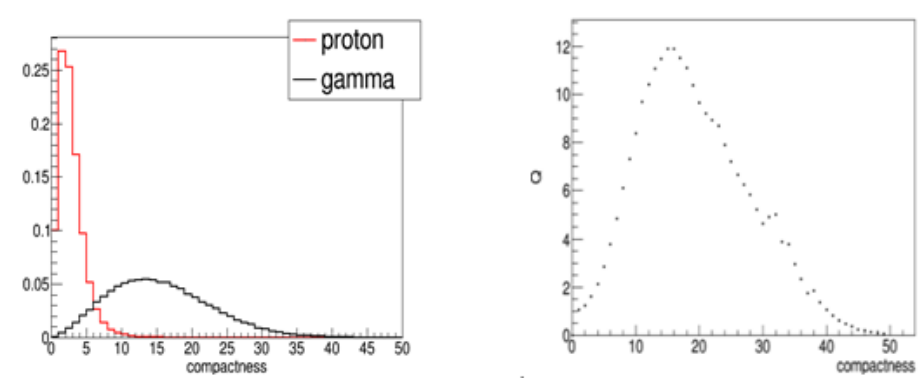

Figure 3: (Left)The compactness distribution of proton and gamma. (Right) The distribution of Q factor.

The result is shown in Fig. 3, the left one is the compactness distribution of gamma and proton, the right one is distribution of $\mathrm{Q}$, a parameter used to measure the level of particle identification.

\subsection{The cosmic ray background Estimation}

The coordinates of Mrk421 in the equatorial system (RA, Dec) are $\left(83.63^{\circ}, 22.02^{\circ}\right)$. Events measured by WCDA-1 within an all-sky map. Then we select an area of $5^{\circ} \times 5^{\circ}$, centered at the Mrk421 direction, have been collected and filled in a grid of $0.1^{\circ} \times 0.1^{\circ}$, after converting the information of the particles from local coordinates to equatorial coordinates. These maps are still exists cosmic-rays background events, we use direct integration to calculate the excess of signals from Mrk421 direction. The method is used based on the assumption that the events of cosmic rays received by detector are isotropic, and the relative receptivity of the detector in all directions can be considered unchanged in a short time if the configuration of the detector does not change greatly.[3] Based on the assumption, a local efficiency map is created by averaging counts over two hours in right ascension around any location.

\section{Results for Mrk421}

\subsection{Flux Light Curve}

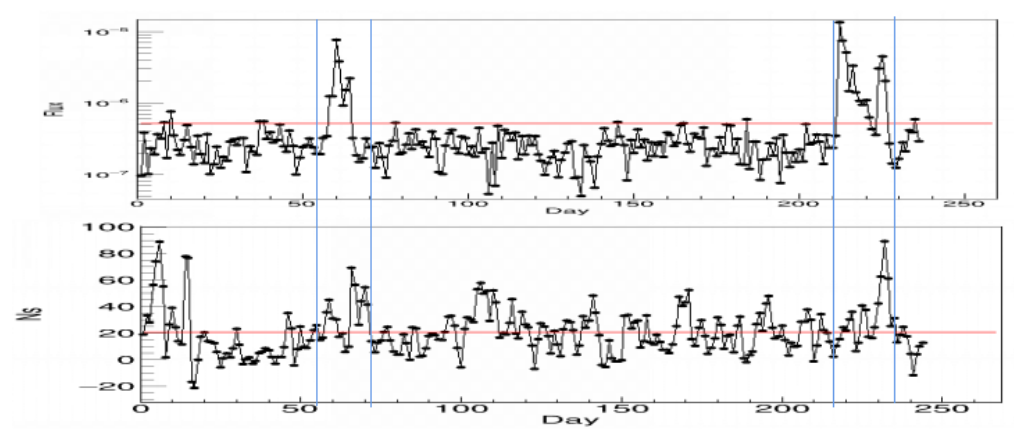

Figure 4: Mrk421 light curves in different bands, from 2019 July 1 to 2020 February 29 . The upper one is the result of Fermi-LAT, each bin of it contains the event every day. The lower one represents the result of WCDA and each bin of it contains the event averaged 3 days.

In this paper, we will focus on the $\mathrm{GeV}$ and $\mathrm{TeV}$ flares, with the aim to investigate the spectral variations at flaring state, compared to the low-activity state. We will define different states of 
activity of Mrk421 based on the light-curves obtained by Fermi-LAT and WCDA. Fig. 4 shows the light curves of Mrk421, the Fermi-LAT cover the range from $100 \mathrm{KeV}-300 \mathrm{GeV}$, while the WCDA covers $\mathrm{TeV}$ energy. According to the eight-months light curves, there exits obvious excess in the flux from 2019 August (MJD=58720) to 2019 September (MJD=58729) and 2, Feb,2020 (MJD=58881) to 20 , Feb, 2020 (MJD=58899), we will define the periods as flaring phase, for a live time of $5.38 \times 10^{6} \mathrm{~s}$. The other days are defined as steady phase, for a live time of $5.44 \times 10^{5} \mathrm{~s}$. From the light curve, we find variability of VHE gamma-ray flux is roughly correlated with $\mathrm{GeV}$ flares[2].

\subsection{The spectral energy distribution of Mrk421}

In this section, we report the SEDs observed by WCDA in the two different states. The event counts sky map and the criteria for data are mentioned in Section 3. To model the spectral energy distribution $\mathrm{f}(\mathrm{E})$, we assume a log-parabolic function

$$
\phi(E)=\phi_{0}\left(\frac{E}{3 T e V}\right)^{-\alpha-\beta \ln \left(\frac{E}{3 T e V}\right)}
$$

Based on the hypothesis of a log-parabolic model, we will obtain the expected number of events $N_{i}^{\text {exp }}$ for each bin each energy range. Then the SED is obtained by minimizing the $X^{2}$ function, where $N_{i}^{o b s}$ is the number of events exceeding the background in the i-th bin j-th energy of $\mathrm{N}_{\text {fitc }}$, $\sigma_{i}^{o b s}$ is the statistical error of the events.

$$
X^{2}=\Sigma_{i=1}^{4} \frac{\left(N_{i}^{o b s}-N_{i}^{e x p}\left(\phi_{0}, \alpha, \beta\right)\right)^{2}}{\left(\sigma_{i}^{o b s}\right)^{2}}
$$

Taking the steady phase as an example, the number of signals from the Mrk421 direction is shown in Fig. 5 for each energy bin with the background cosmic rays subtracted, be fitted well with a 2-dimension Gaussian functional template.

The corresponding significance, number of events exceeding the background and the angular resolution in each bin of the two different source states are listed in Table 1 (Left represents the steady phase and right represents the flaring phase).

The SED measured by WCDA-1 is shown in Fig. 6. The energy of each point is the gamma-ray median energy for the corresponding Nfitc interval. The red points represents the steady phase, while the blue points represents the flaring phase. The spectral parameters of the two states obtained by logarithmic parabola fitting are shown in Table 2, The results show that there is no obvious difference in the energy spectrum distribution between the two periods.

\begin{tabular}{|c|c|c|c|c|c|c|c|}
\hline $\mathrm{N}_{\text {fitc }}$ & $\mathrm{E}_{\text {med }}(\mathrm{TeV})$ & Excess & sig & $\mathrm{N}_{\text {fitc }}$ & $\mathrm{E}_{\text {med }}(\mathrm{TeV})$ & Excess & sig \\
\hline $60-100$ & 0.62 & 1382.62 & 9.9 & $60-100$ & 0.60 & 122.45 & 4.2 \\
\hline $100-200$ & 1.45 & 821.50 & 16.3 & $100-200$ & 1.41 & 98.90 & 5.8 \\
\hline $200-300$ & 3.41 & 214.11 & 11.7 & $200-300$ & 3.34 & 9.70 & 3.1 \\
\hline $300-800$ & 7.27 & 57.17 & 7.4 & $300-800$ & 7.11 & 20.84 & 6.9 \\
\hline
\end{tabular}

Table 1: Summary of data used in the measurement of SED of Mrk421 

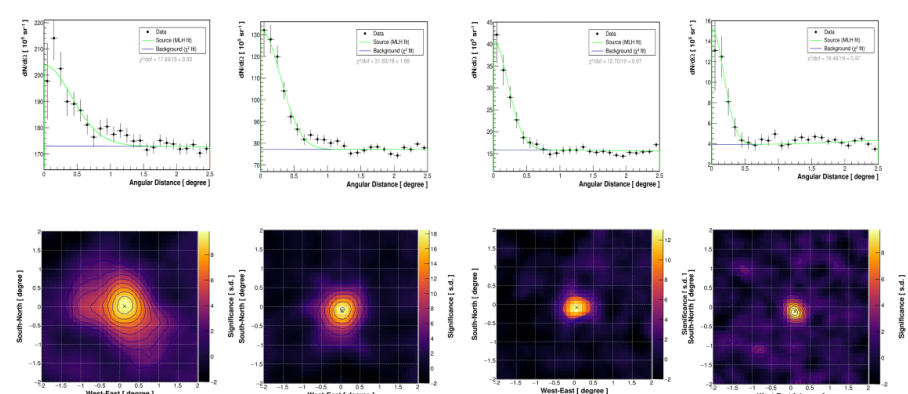

Figure 5: The upper four are signals for different energy range, namely [60,100], [100,200], [200,300] and $[300,800]$ from left to right. The lower four are the corresponding significance map.

\begin{tabular}{|c|c|c|}
\hline phase & $\phi_{0}\left(\mathrm{TeV}^{-1} \mathrm{~cm}^{-1} \mathrm{~s}^{-1}\right)$ & $\alpha$ \\
\hline Steady phase & $4.80 \pm 0.37$ & $3.13 \pm 0.092$ \\
\hline Flaring phase & $4.13 \pm 1.13$ & $3.18 \pm 0.28$ \\
\hline
\end{tabular}

Table 2: The result of different phases on Mrk421

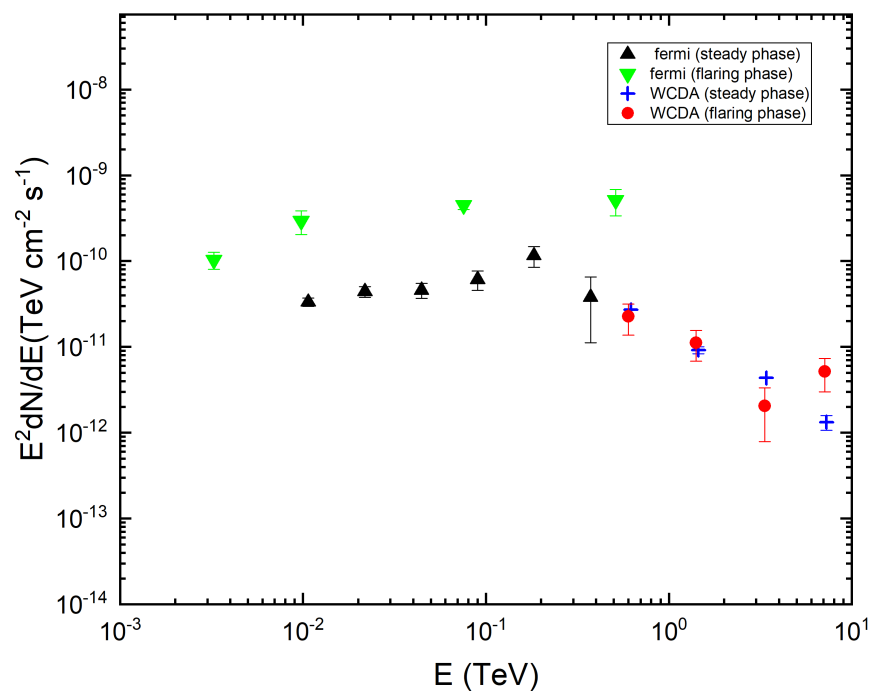

Figure 6: The SED of the Mrk421

\section{Summary and Outlook}

In this paper, we presented the light curves for Mrk421 with the eight months of data from the July, 2019 to February, 2020, a period that includes both steady states and episodes of strong flaring activity. Due to the WCDA and Fermi-LAT data, the observation concerns GeV and TeV energy bands. The main results of this work can be summarized as follows.

(1) The flux variation in $\mathrm{GeV}$ and $\mathrm{TeV}$ bands was analyzed, we find the $\mathrm{GeV}$ gamma-ray flux appears to be roughly correlated with $\mathrm{TeV}$ gamma-ray flux.

(2) Mrk421 showed both low and high activities in this period. According to the observed light curves we divided this period into steady phase and flaring phase, it is found that there is no distinct 
difference in the energy spectrum index between the two periods. The energy index in the steady phase is -3.13 and in the flaring phase is -3.18 .

(3) In the next work, we will use the full array of data and increase the data sample, and divide the flaring period more carefully according to different flux variation to complete the measurement of Mrk421 energy spectrum distribution.

\section{Acknowledge}

This research work is also supported by the following grants: the National Science Foundation of China (NSFC) (Grant Nos.11775131 and U 1931108).

\section{References}

[1] Randall L. Oglsby, Modeling the spectral Energy Distribution of Mrk421. (2013)

[2] B. Bartoli, et al. , The Astrophysical Journal Supplement Series, 222:6 (17pp), 2016 January.

[3] MILAGRO Collaboration, APJ, 595 (2) (2003) 803-811. 